\title{
The impact of stochastic parametrisations on the representation of the Asian summer monsoon
}

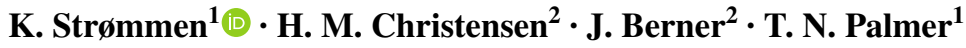

Received: 15 December 2016 / Accepted: 1 June 2017 / Published online: 20 June 2017

(c) The Author(s) 2017. This article is an open access publication

\begin{abstract}
The impact of the stochastic schemes Stochastically Perturbed Parametrisation Tendencies (SPPT) and Stochastic Kinetic Energy Backscatter Scheme (SKEBS) on the representation of interannual variability in the Asian summer monsoon is examined in the coupled climate model CCSM4. The Webster-Yang index, measuring anomalies of a specified wind-shear index in the monsoon region, is used as a metric for monsoon strength, and is used to analyse the output of three model integrations: one deterministic, one with SPPT, and one with SKEBS. Both schemes show improved variability, which we trace back to improvements in the El Niño-Southern Oscillation (ENSO) and the Indian Ocean Dipole (IOD). SPPT improves the representation of ENSO and through teleconnections thereby the monsoon, supporting previous work on the benefits of this scheme on the model climate. SKEBS also improves monsoon variability by way of improving the representation of the IOD, in particular by breaking an overly strong coupling to ENSO.
\end{abstract}

Keywords Asian monsoon - Stochastic parametrisations . Webster-Yang Index · Interannual variability

\section{Introduction}

The difficulty of accurately representing the Asian summer monsoon in a climate model is well known (e.g. Sperber

K. Strømmen

kristian.strommen@physics.ox.ac.uk

1 Clarendon Laboratory, Physics Department, AOPP Building, Parks Road, Oxford OX1 3PU, UK

2 NCAR, Boulder, CO 80307-3000, USA and Palmer 1996; Kang et al. 2002; Annamalai 2007). This is largely because, while the basic underlying mechanism is relatively simple, being driven by a land-sea thermal gradient developing during boreal summer, the relative regional intensity of the monsoon depends sensitively on multiple local and global factors (Sperber and Palmer 1996; Ju and Slingo 1995). Since Walker (1923), one of the most significant global factors influencing the monsoon has been recognized as the El Niño-Southern Oscillation (ENSO). Roughly, years with a weak monsoon (measured e.g. in terms of All India Rainfall) tend to coincide with El Niño years, and years with strong monsoons to La Niña (Webster and Yang 1992; Ju and Slingo 1995). However, the correlation is not decisive. Indeed, El Niño years have seen almost the full range of possible Indian rainfall at various points in the last hundred years, from drought to excessive rains (Krishna Kumar et al. 2006). Averaging over longer timescales reveals a stronger correlation, but even this correlation is subject to variation and has lately been observed to be in decline (Krishna Kumar et al. 1999). Arguably the main local factor influencing the impact of the ENSO teleconnection is the Indian Ocean Dipole (IOD), an oscillation of sea-surface temperatures between the western and eastern regions in the Indian Ocean associated with a localized Walker cell (Saji et al. 1999). A positive IOD (anomalous warming in the western basin) tends to weaken the monsoon while a negative event (anomalous warming in the eastern basin) strengthens it (Ashok et al. 2001; Yamagata et al. 2004). While IOD events can be triggered by ENSO, they often occur independently, and have been shown to influence the monsoon as a stand-alone phenomenon (Yamagata et al. 2004; Meyers et al. 2006). Thus whether or not the IOD is in phase with ENSO can have a significant impact on the observed monsoon intensity in a given year (Ashok et al. 2001). 
In order for a GCM to accurately simulate the interannual variability of the monsoon, it is therefore crucial to accurately represent ENSO, the IOD, their interactions, and impacts. Multi-model comparison studies (Sperber and Annamalai 2013) have shown that many Coupled-ModelIntercomparison-Project-5 (CMIP5) models still struggle to capture these processes, and display several systematic deficiencies in monsoon representation. In general, when it comes to representing multiple aspects of the climate, many models are both over-confident, due to lack of spread, and suffer from systematic biases in mean states. One approach to alleviate this problem is to introduce a stochastic noise scheme, which aims to represent the variability inherent in unresolved sub-grid scale processes (Palmer et al. 2009). This technique is now wide-spread in medium-range and seasonal weather forecasts, where they have a beneficial effect on the spread as well as the mean-state in predictions (Palmer et al. 2009; Weisheimer et al. 2014; Williams 2012). More recently, the potential benefits of a particular stochastic scheme on the actual climate of a model has been examined (Berner et al. 2008). In particular, a recent study (Christensen et al. 2016) considered the impact of stochastic schemes on ENSO in a coupled GCM. They found a significant improvement in both the periodicity and amplitude of ENSO compared to the purely deterministic model. In this paper, we will consider the impact on the Asian summer monsoon. In particular, we will focus on interannual variability, and the impact thereon by the two most commonly used stochastic schemes, the Stochastically Perturbed Parametrisation Tendencies (SPPT) and the Stochastic Kinetic Energy Backscatter Scheme (SKEBS).

The paper is structured as follows. In Sect. 2 we outline the model set-up, the stochastic schemes used, data processing conventions and observational data used. We also define the main diagnostics utilized to measure monsoon strength. The focus will be on monsoon winds, utilizing the Webster-Yang index (Webster and Yang 1992), an index designed to capture the more large-scale monsoon-ENSO teleconnection. In Sect. 3 we present the main results, and discuss them in Sect. 4. Final conclusions are drawn in Sect. 5. An Appendix is included for some additional discussion.

\section{Model set-up, diagnostics and observational data}

\subsection{Model set-up}

The model integrations were performed using the oceanatmosphere coupled Community Climate System Model Version 4, (CCSM4; see Gent et al. 2011) with an atmosphere resolution of $0.9^{\circ} \times 1.25^{\circ}$ and a $1^{\circ}$ ocean with

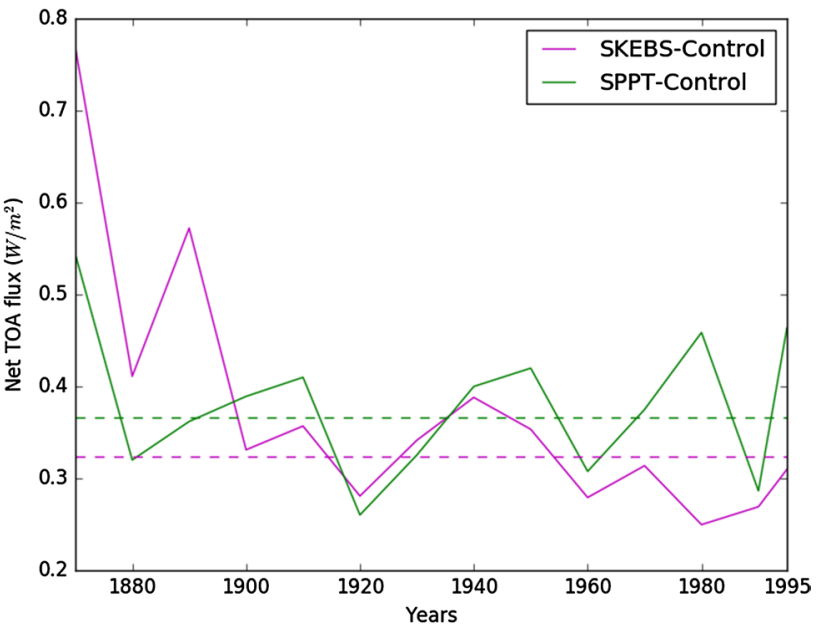

Fig. 110 year averages of net TOA flux differences. The y-values show the average for the decade following the corresponding year (so the point at 1880 shows the average over 1880-1890). The stipled lines show the averages over the period 1900-2000, with matching colors

historical forcings. The integrations cover the period 1870-2004. Three runs were performed, one deterministic control, one with the SPPT scheme and one with SKEBS. These runs will be referred to, respectively, by the shorthand titles Control, SKEBS and SPPT. The initial conditions were obtained from an 850 year spin-up of the deterministic model with pre-industrial forcing.

We expect that the stochastic schemes will lead to a change in the modelled climate, and that the model will require an additional spin-up time for the atmosphere to equilibrate with the ocean. Furthermore, no additional tuning was performed after implementing the stochastic schemes. By keeping the underlying physical parameters the same as for Control, this ensures the raw impact of the stochastic schemes can be determined. However, it is possible that, as a result, the flux balance at top of atmosphere will be affected, leading to long-term spurious climate drifts in time. Both the model spin-up and climate balance must be assessed before the data is analysed.

Figure 1 shows the difference in net radiative fluxes (shortwave minus longwave) at the top of the atmosphere (TOA) for the two schemes for different decades. The initial shock to the atmosphere caused by the schemes, especially SKEBS, is visible, before the schemes appear to settle down to a relatively constant, positive difference from around 1900 onwards. Table 1 shows the differences in TOA fluxes over the first and second half of the 20th century, which are seen to be virtually the same. ${ }^{1}$ We consider

\footnotetext{
${ }^{1}$ Note that this relatively constant difference of around 0.3 for each scheme, while significant, is relatively small. However, it must all in all be considered a slight degradation, as the deterministic model
} 
Table 1 Globally averaged net TOA-flux differences over different timeperiods

\begin{tabular}{lll}
\hline & SKEBS-CTRL & SPPT-CTRL \\
\hline 1900-1950 & 0.32 & 0.36 \\
$1950-2000$ & 0.30 & 0.36 \\
\hline
\end{tabular}

this as evidence that the atmospheric drift introduced by the schemes has stabilized after the first 30 years. By discarding this period for our analysis we can thereby focus on the raw impact of the schemes themselves. The impact of any potentially minor remaining drift is considered in an Appendix: we consider it to be negligible. In the paper we will generally consider the two time-periods 1900-2000 and 1979-2010 to compare with observations.

The SPPT scheme perturbs the total physics tendency at each time-step to account for unresolved sub-grid scale variability. The noise is multiplicative of the form $1+\epsilon$ where $\epsilon$ is drawn from a randomly generated spectral pattern which evolves in time as an AR(1) process, with a decorrelation time of 6 hours and a horizontal decorrelation scale of $500 \mathrm{~km}$. For more details see Christensen et al. (2016) or Palmer et al. (2009).

The SKEBS scheme aims to represent model uncertainty arising from unresolved sub-grid scale processes by introducing random perturbations to streamfunction and potential temperature tendencies. SKEBS is based on the rationale that a small fraction of the model dissipated energy interacts with the resolved-scale flow and acts as a systematic forcing. Originally developed for Large Eddy Simulations (Mason and Thomson 1992), it was adapted to numerical weather prediction by (Shutts 2005). Temporal correlations are introduced into the noise by evolving the spectral coefficients $f_{n}^{m}$ of the tendencies as an $\operatorname{AR}(1)$ process:

$f_{n}^{m}(t+\Delta t)=\alpha f_{n}^{m}(t)+\sqrt{(\alpha-1)} g_{n} \epsilon(t)$,

where $\alpha$ is the linear autoregressive parameter determining the temporal decorrelation time, $g_{n}$ the wavenumberdependent noise amplitude and $\eta$ a Gaussian white-noise process with mean zero and variance $\eta$. The amplitude of the noise $g_{n}$ evolves according to the rule $g_{n}=b n^{p}$. This process is done independently for both the streamfunction and potential temperature tendencies. The behavior of this

\section{Footnote 1 (continued)}

tends to have high fluxes already. For example, in the period 2000 2004, Control has a net TOA of around $0.96 \mathrm{~W} / \mathrm{m} 2$, while observational estimates (Trenberth et al. 2009) put it at $0.9 \pm 0.15 \mathrm{~W} / \mathrm{m}^{2}$. This also suggests the difference is greater than observational uncertainty. scheme is determined by the following parameters: the exponent of the power law $p$, the wavenumber perturbation range, and the amplitude of forcing energy (which determines $b$ ). For the results presented here, all wavenumbers were perturbed and the power law exponents chosen to be $-14 / 3$ and -3 for the streamfunction and temperature forcing, respectively. Note that a choice of 14 / 3 for the streamfunction forcing leads to a slope of $-5 / 3$ for the perturbation kinetic energy. No strong sensitivity to the forcings slopes was found.

Different from the original implementation in the ECMWF model (Shutts 2005; Berner et al. 2009), the total instantaneous dissipation rate, which determines the amplitude of the perturbation, is here assumed to be spatially and temporally constant, resulting in a state-independent (additive) stochastic forcing. This simplification relies on the underlying model dynamics to determine which perturbations will grow and which ones will be damped. On longer timescales, a state-dependent forcing amplitude is presumably desirable, but the estimation of the dissipation rates complicated by the fact that they will depend on the dynamical core and physical parameterization schemes, as well as on the numerical resolution. Hence, in the interest of simplicity, the dissipation rates are assumed constant. Their amplitudes were chosen small enough, so that the potential and kinetic-energy slopes of the total fields are unchanged, but as large as possible otherwise, in order to see the maximal impact of the stochastic scheme. In order not to continously inject potential and kinetic-energy into the model, the SKEBS tendencies were treated as all other physical-tendencies and sent through the so called "energy fixer", which adds a uniform increment to the temperature field to compensate for the global average energy lost by the dynamical core that time step.

\subsection{Observational data}

We use the ECMWF re-analysis dataset ERA-Interim (Dee et al. 2011), covering the period 1979-2010, both for SSTs and wind-shear spatial patterns and means. However, to bolster confidence in the analysis, we will also use the longer ERA-20C (Poli et al. 2016) re-analysis when computing various power spectra, which covers the period 1900-2000. The spectra were in all cases also computed over the period 1979-2010 and compared to ERA-Interim: the results were consistent with the longer interval. For rainfall, we used GPCP data (Adler et al. 2003) to look at the period $1979-2010 .^{2}$

\footnotetext{
${ }^{2}$ GPCP Precipitation data was provided by the NOAA/OAR/ESRL PSD, Boulder, Colorado, USA, from their web site at http://www.esrl. noaa.gov/psd/.
} 


\subsection{Diagnostics}

To measure the large-scale wind circulation in the monsoon, we follow (Webster and Yang 1992) in considering a zonal 'wind-shear index', measured as zonal wind anomaly differences between 850 and $200 \mathrm{hPa}$, as our main variable, i.e.

$U_{850}^{*}-U_{200}^{*}$

where $U_{n}^{*}$ denotes the zonal wind anomaly at pressure level $n$. The anomaly is calculated relative to the average over the period 1900-2000 when compared to ERA-20C, and over the period 1979-2000 when compared to ERA-Interim. When averaged over the region $0 \mathrm{~N}-20 \mathrm{~N}, 40 \mathrm{~W}-110 \mathrm{~W}$ in space, and over the monsoon period in time, we obtain the Webster-Yang index $W Y(t)$. This will be our choice of metric for measuring the strength of the monsoon in a given year.

We also considered total precipitation during the monsoon period in the same region (as well as restricted to a rectangle covering India), as discussed in Sect. 3.4. The results obtained using this rainfall metric are very similar to those obtained using the WY-index, so with the exception of that section, the paper will focus on the the WY-index.

In observational data, the monsoon summer period is defined as the months June-July-August-September (JJAS). However, as shown in Sect. 3.1, the model has a systematic error producing a delayed monsoon. In order to not penalize the model repeatedly for this, the months July-August-September-October (JASO), the period in the model with maximal rainfall/WY-index, will be considered as the monsoon period when diagnosing model output. Note that delayed onset is a common error amongst GCMs (Sperber and Annamalai 2013).

To examine ENSO, we use the Nino 3.4 index, i.e. detrended monthly anomalies of sea-surface temperatures (SSTs) in the region 5S-5N, 190E-240E.

The IOD, first discovered by Saji et. al (1999) is characterised as an oscillation of SST's between the western and eastern Indian ocean. A positive (negative) IOD is characterized by an anomalous warming (cooling) in the west and an anomalous cooling (resp. warming) in the east. The dynamics of the IOD have been comprehensively studied and documented in Yamagata et al. (2004) (see also: Meyers et al. 2006; Ashok et al. 2004). We will measure the IOD using the Dipole Mode Index (DMI) from Saji et al. (1999), defined as the difference in monthly SST anomalies between the western region $10 \mathrm{~S}-10 \mathrm{~N}, 50 \mathrm{E}-70 \mathrm{E}$ and the eastern region $10 \mathrm{~S}-0 \mathrm{~N}, 90 \mathrm{E}-110 \mathrm{E}$, where we remove both the annual cycle and any linear trends from global warming. Some recent work suggests measuring the IOD as the leading EOF of detrended Indian Ocean SST anomalies

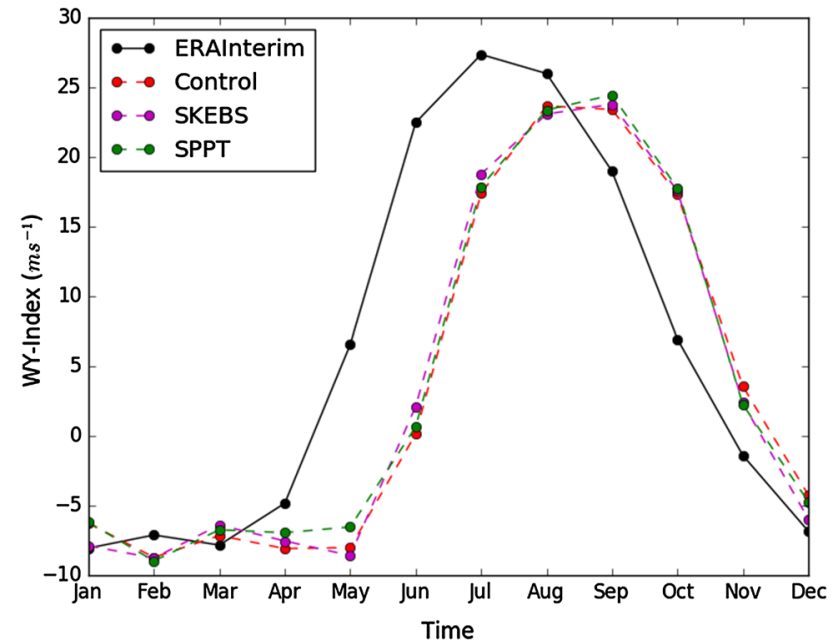

Fig. 2 Monthly climatological values of the WY-index over the period 1979-2010, showing the monsoon progression

averaged over the period September-October-Novem$\mathrm{ber}^{3}$ (SON) rather than with the DMI, to better capture the potential variations of the IOD spatial pattern in different models (Meyers et al. 2006). However, in CCSM4, the principal EOF spatial pattern associated with this quantity (see Fig. 8) is well captured by the DMI already, so for simplicity we use the DMI instead.

All figures were produced using Python 2.7 (details available on request).

\section{Results}

\subsection{Impact on the WY-index}

There is a systematic onset error in CCSM4, which is not alleviated by either stochastic scheme. This is clearly seen in Fig. 2, showing the monthly climatology of the WYindex. It can be seen that while the model does produce a monsoon which captures the steep ascent/descent reasonably well, it starts roughly one month later than observed. It is also clear that the model generally has too weak a monsoon circulation. The same delayed onset is visible also when considering India rainfall.

It is perhaps not surprising that the stochastic schemes are unable to resolve this error. The monsoon, in contrast to phenomena like ENSO, is driven by a seasonal process, the onset of which is entirely dependent on the development of a persistent land-sea thermal gradient in the summer months, and is therefore unlikely to be triggered earlier by means of random perturbations with small time-scales.

\footnotetext{
3 This is the period when the IOD typically peaks.
} 
Fig. 3 Means of the wind-shear index $(\mathrm{m} / \mathrm{s})$ during monsoon period, averaged over the years 1979-2010 for a ERA-Interim, b Control, $\mathbf{c}$ SKEBS and d SPPT. The value $M$ is the average over the whole region (a) $M=23.7$

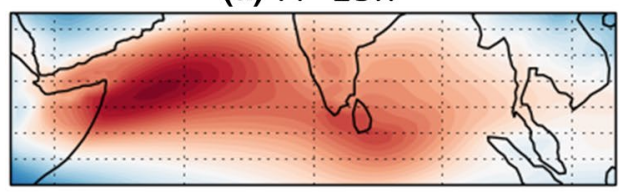

(c) $M=20.8$

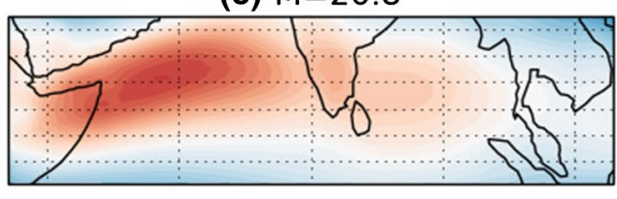

(b) $M=20.4$

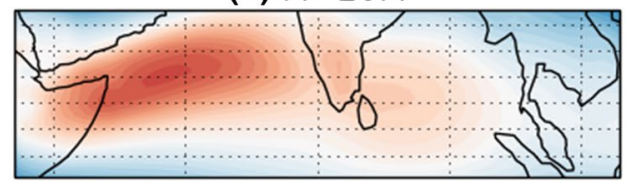

(d) $M=20.9$

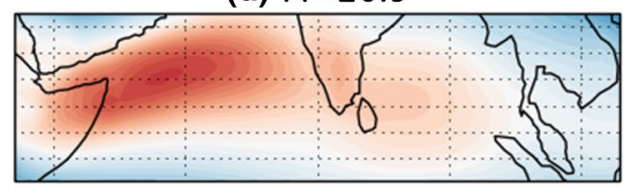

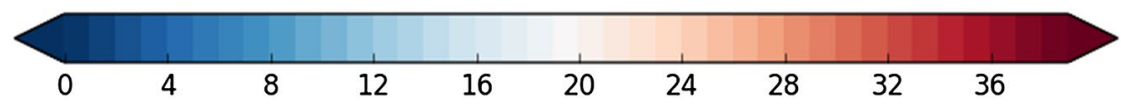

Fig. 4 Means of $U_{850}$ during the monsoon period, averaged over the years 1979-2010; a ERAInterim, b Control-ERAInterim, c SKEBS-Control and d SPPTControl. $E_{a b s}$ denotes the mean absolute error of each scheme (deterministic, SKEBS and SPPT) compared to observations in each case as well. Plot $\mathbf{b}$ shows the model bias of Control compared to observations as shown in a. Plots $\mathbf{c}, \mathbf{d}$ show how SKEBS and SPPT change this bias, and should be compared to $\mathbf{b}$ : if the color at a gridpoint in $\mathbf{c}$ or $\mathbf{d}$ is opposite (the same) to that in $\mathbf{b}$, the scheme is reducing (enhancing) the bias at that gridpoint. The total impact on the Control-ERA bias can be estimated by $E_{a b s}$ (a)

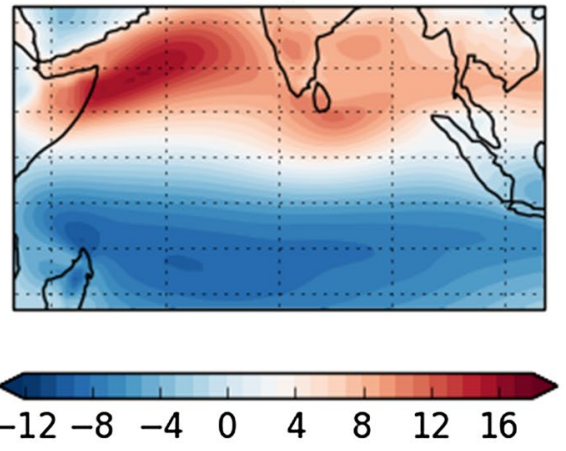

(c) $\left(E_{a b s}=1.42\right)$

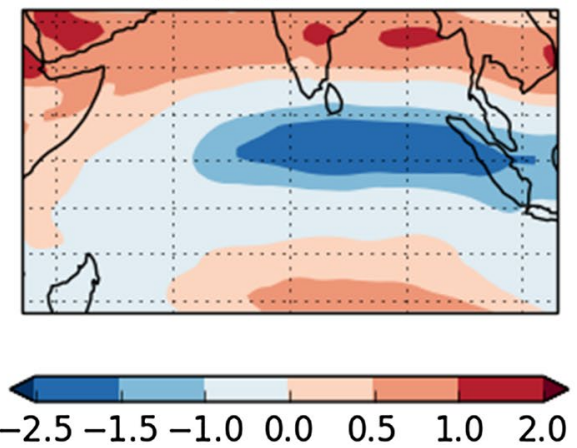

(b) $\left(E_{a b s}=1.00\right)$
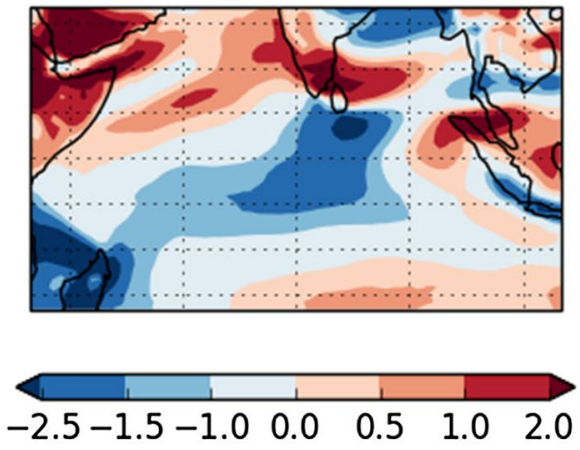

(d) $\left(E_{a b s}=1.16\right)$

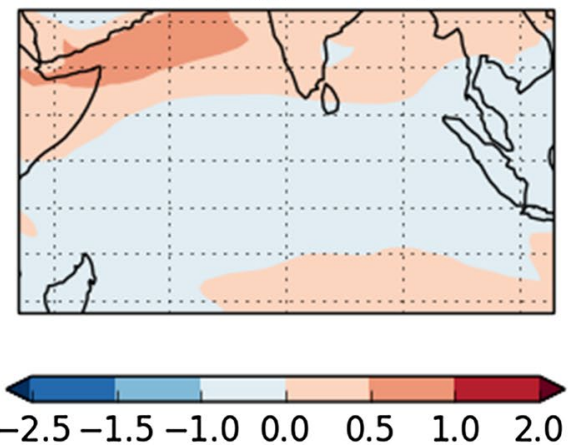

This indicates that stochastic schemes, unless very carefully constructed, cannot be expected to overcome more fundamental model errors.

Figure 3 shows spatial maps of the zonal wind-shear index. In all simulations this index is too small compared to observations across the whole region, though most notably near Sri-Lanka. The main source of this difference stems from the fact that the deterministic model has a monsoon circulation which is overly strong. Figure 4 shows both the mean $U_{850}$ for ERA-Interim in (a), as well as difference plots showing the bias of Control compared to ERA-Interim in (b) and the changes in this bias introduced by SKEBS, (c), and SPPT, (d). The figure also includes the absolute error $E_{a b s}$ of each model simulation compared to observations. Figure $4 \mathrm{a}$ shows the observed overturning circulation characteristic of the monsoon, with easterly winds hitting the coast of east-Africa and deflecting back towards India as part of the Findlater jet. The difference plot, $b$ shows that Control has increased the strength of both the westward and eastward moving components of the circulation, with the greatest change being in increasing the eastward flow. This reduces the $U_{850}$ anomaly leading to a reduced WY-index. 


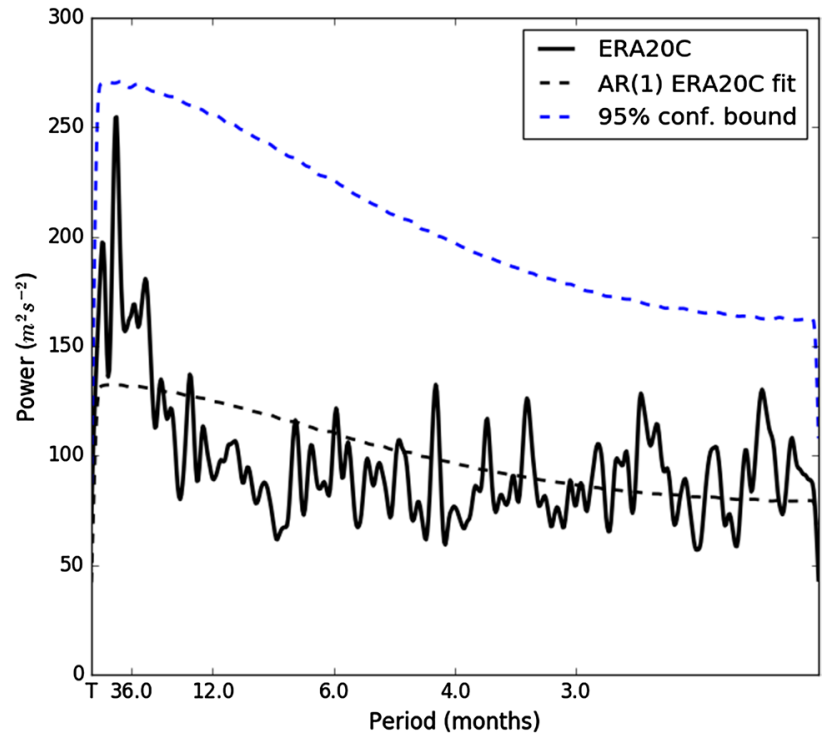

Fig. 5 Power spectrum of WY-index for ERA20C over the period 1900-2000, along with the best AR(1) fit and its upper 95\% confidence bound (smoothed using a Hanning window with width 14). Power falling within this interval is considered to be statistically comparable to ERA20C. Note that the seasonal cycle has been removed

It can be seen in Fig. 4d that SPPT has only a small impact on the circulation compared to Control, somewhat enhancing the Findlater jet, while in (c), SKEBS has amplified the bias by more: this is also reflected in the increase in $E_{a b s}$ for both SKEBS and SPPT. The difference between the datasets in the upper circulation at $200 \mathrm{hPa}$ is negligible.

By contrast, a big difference is observed in interannual variability, measured via the power spectrum. To estimate statistical significance for these spectra, we fit an $\operatorname{AR}(1)$ process to the monthly WY-index of ERA-20C, with the seasonal cycle removed, and generate an estimate of its spectrum. Confidence intervals are then generated relative to this AR(1) model in the following manner: we fit an $\mathrm{AR}(1)$ process to the monthly index and generate 10,000 simulations, providing a probability distribution of potential power at each frequency. The percentiles are obtained by taking, at each frequency, the power associated to two or three standard deviations from the mean. The full power spectrum for ERA-20C, with confidence intervals, can be seen in Fig. 5. Power at a given frequency falling within the 95\% (or 99\%) confidence interval is considered to be statistically close to ERA-20C with respect to the confidence interval in question.

The power spectra of the models can be seen in Fig. 6, where we have for simplicity restricted the range to periods on interannual timescales: this amounts to zooming in on the left-most part of the full spectrum as seen in

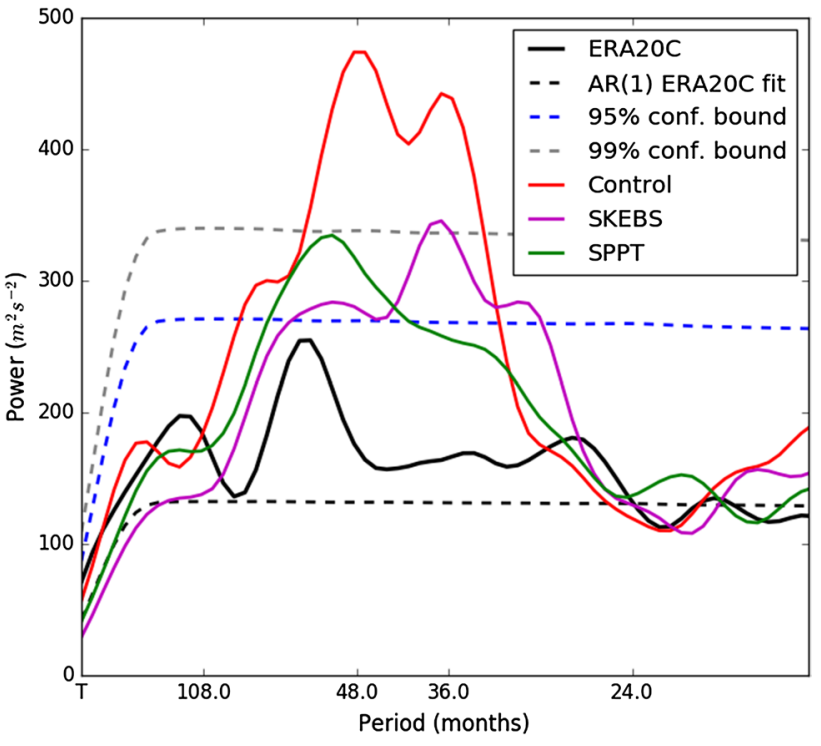

Fig. 6 Power spectrum of monthly WY-index over the years 19002000 (smoothed using a Hanning window with width 14). The confidence bounds are relative to the AR(1) fit of ERA20C

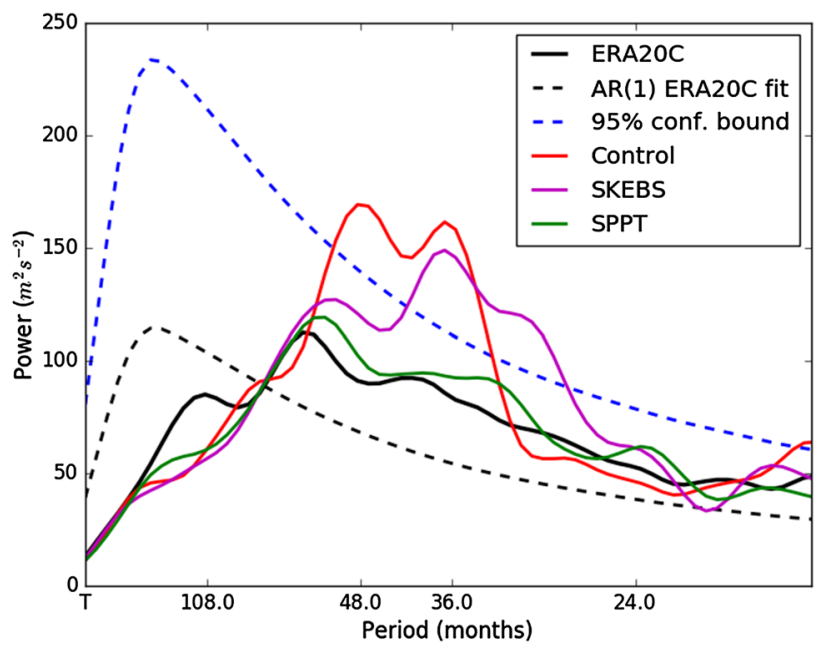

Fig. 7 Power spectrum of the Nino 3.4. index over the years 19002000 (smoothed using a Hanning window with width 14). The confidence bound is relative to the AR(1) fit of ERA20C. Power falling within this interval is considered statistically comparable to ERA20C

Fig. 5. We see that the spectrum of the deterministic run has excessively large peaks at 3 and 4 years compared to reanalysis, which has no notable amount of power at these frequencies. It can be seen that both the SPPT and SKEBS schemes notably improve this bias, though in both cases the power at periods of 3 and 4 years is still unnaturally large. Applying the same statistical test described above, it can be shown that the difference in power between Control and 
Fig. 8 EOF spatial patterns of detrended Indian Ocean SSTs during SON, over the period 1979-2010 ( $\left.{ }^{\circ} \mathrm{C}\right)$ : a ERAInterim, b Control, c SKEBS and d SPPT. Here 'amp' denotes the amplitude $\left({ }^{\circ} \mathrm{C}\right)$; 'frac' the fraction of variance accounted for by this pattern; 'eig' the absolute value of the associated eigenvalue; 'var' the total anomaly variance of the data (i.e. the sum of all eigenvalues), so that frac $=$ eig/ var. The outlined regions are the western and eastern regions used to define the Dipole Mode Index (a): $\mathrm{amp}=0.16$, eig $=105.19$,

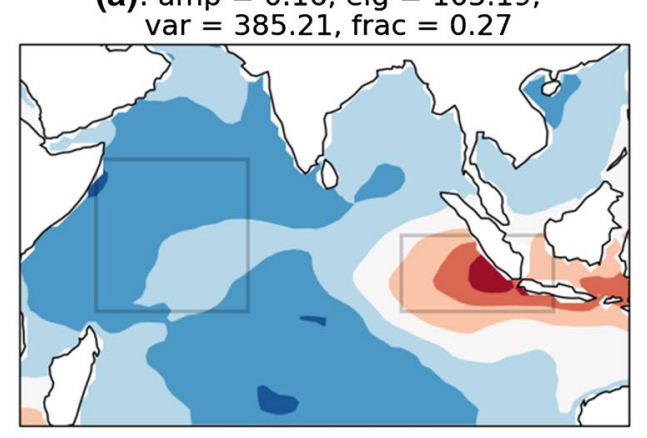

(b): amp $=0.34$, eig $=418.59$,

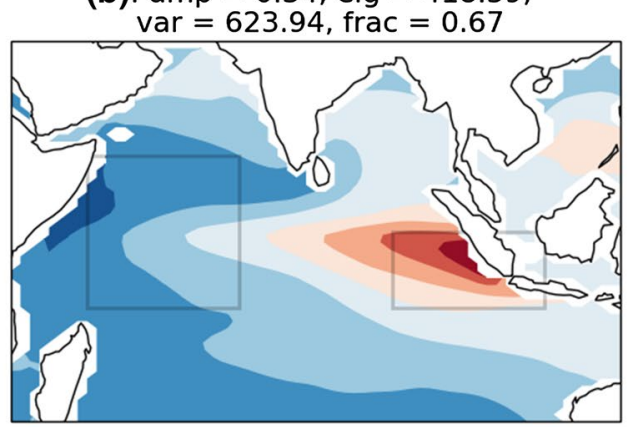

(c) : $\mathrm{amp}=0.17$, eig $=134.89$, var $=304.01$, frac $=0.44$

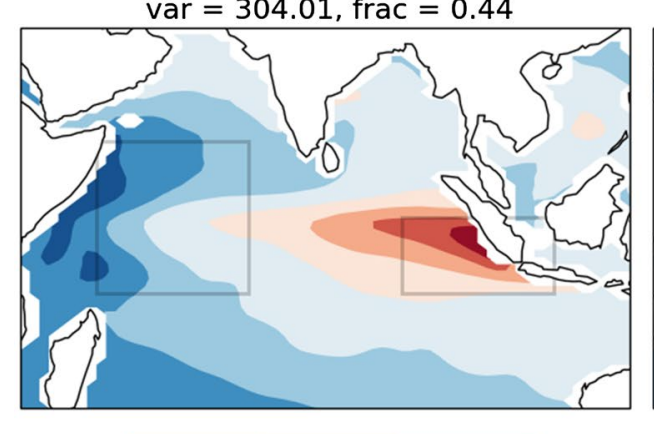

(d): $\operatorname{amp}=0.28$, eig $=342.79$, $\operatorname{var}=532.66$, frac $=0.64$

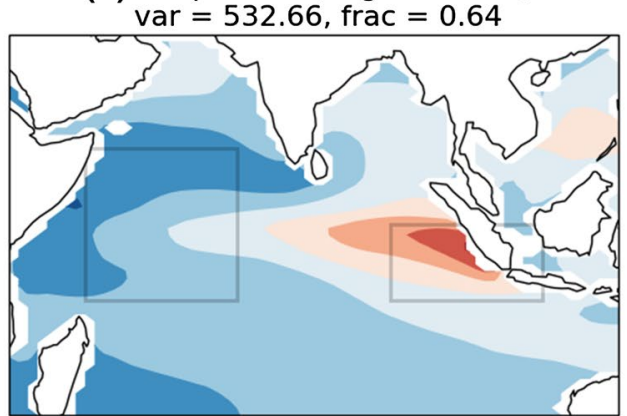

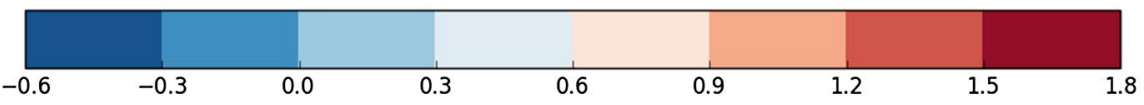

either stochastic scheme is significant with respect to the $95 \%$ confidence bound. ${ }^{4}$

\subsection{Impact on ENSO}

The impact of the SPPT scheme on ENSO was examined and documented extensively in Christensen et al. (2016), where it was stated that SKEBS did not have any notable impact: SKEBS was therefore not considered in that paper. As we will be considering SKEBS here, we will for completeness reproduce the power spectrum of the Nino 3.4. index here, including both schemes. Figure 7 shows the

\footnotetext{
${ }^{4}$ It is possible the reader will be puzzled at the seeming differences in the slopes of the AR(1) fits and confidence intervals in the various spectra shown. We point out here that there is a lot of scope for 'optical illusion'! The confidence interval in Fig. 5 appears much more sloped than that in Fig. 7, which appears almost constant. Note however that in Fig. 6, if one considers the slope of the AR(1) fit and confidence interval in the part of the plot well ahead of the 12 month period mark, these slopes do in fact appear nearly flat, consistent with what is seen in Fig. 7. In the spectrum of Nino 3.4, as seen in the next section, the slope is however much more clearly visible. This is simply due to the fact that for this spectrum, the power is much more concentrated in the interannual region, so the difference in power between this region and higher frequencies is greater, leading to a steeper slope. For the WY-index and the IOD index, more power is found in higher frequencies as well, leading to a less steep slope.
}

result of this computation, verifying the claims of Christensen et al. (2016).

SPPT significantly reduces the excessive peaks at $3 / 4$ years present in the deterministic model and matches ERA$20 \mathrm{C}$ very well. SKEBS appears to have shifted the peak intensity from the range 36-48 months to 30-36 months, though it is hard to estimate if the reduction in the peak at 48 months is significant or not. Indeed, the location of the peak power in Control is not constant through the 20th century, moving more from the 48-month period to the 36-month period over time. Whether this is due to natural variability or the impact of forcing is unclear, but in either case we argue that the shift from SKEBS cannot easily be seen as a significant improvement over Control: it still suffers from an ENSO which is 'overly periodic' in the sense of having a too large spectral peak.

\subsection{Impact on the IOD}

The IOD is usually either defined via the DMI or as the leading EOF of detrended Indian Ocean SST anomalies averaged over the period SON. Figure 8 shows this EOF for observations and the three model runs. The EOF has been scaled by multiplying it with the square-root of the associated eigenvalue, so that the scale in the figure is in units of Celsius. The rectangles show the western and eastern 'pole' 
used in the definition of the DMI. It can be seen that while there are spatial differences in the different simulations, the DMI regions still broadly capture the EOF pattern. Manual inspection of the SST data also showed that the EOF pattern captures the typical IOD pattern that actually occurs in each simulation. Therefore we will use both the leading EOF and DMI to measure the IOD.

Following Weller and Cai (2013), we measure the amplitude (units of Celsius) of the IOD as the standard deviation of the EOF spatial pattern (scaled as indicated). We also compute the total fraction of variability that the IOD accounts for. For completeness, we also computed the absolute value of the eigenvalue associated to this EOF, which measures the magnitude of variance it accounts for, as well as the total variance itself..$^{5}$ The results are included in Fig. 8. One can see that the deterministic model has too large an amplitude (around two times that of observation), and a too high fraction of the SST variance is accounted for by the IOD (around two thirds compared less than a third). ${ }^{6}$ Indeed, in the deterministic model, the total variability is notably higher than in observations, and the amount of variability in Control coming from the IOD alone, as measured by the associated eigenvalue, is greater than the total variability in observations. SPPT also has far too great a total variability, and the IOD is still accounting for far too much variability, both in absolute terms and in terms of fraction of variability. The amplitude is also still excessive.

On the other hand, SKEBS has shifted the amplitude and variability of the IOD to be extremely close to observations. However, the total variability has also decreased to a value which is somewhat too low (though still much closer to observations), meaning the IOD is still accounting for too great a fraction of the variability, though less so than Control and SPPT.

Figure 9 shows the power spectrum of the IOD, measured now by the DMI. The deterministic model has an excessively large peak at 4 years, compared to observations which appear as a generic red-noise process with no particular power at any one frequency. Clearly SKEBS is a major improvement in this regard, having entirely eliminated this excess power. SPPT has shifted the spectral peak to 3 years, but still appears to be excessively periodic

\footnotetext{
5 That is, the weighted trace of the covariance matrix of the centered data-matrix obtained from the SST anomalies. Equivalently, this is the sum of the EOF eigenvalues. Note that the EOF vectors were computed to have length 1 , which is why the eigenvalues are so much larger than the spatial scales.

${ }^{6}$ It follows from this that while in observations, total variability is split between several EOF's, less are required in Control to account for the majority of the total variability.
}

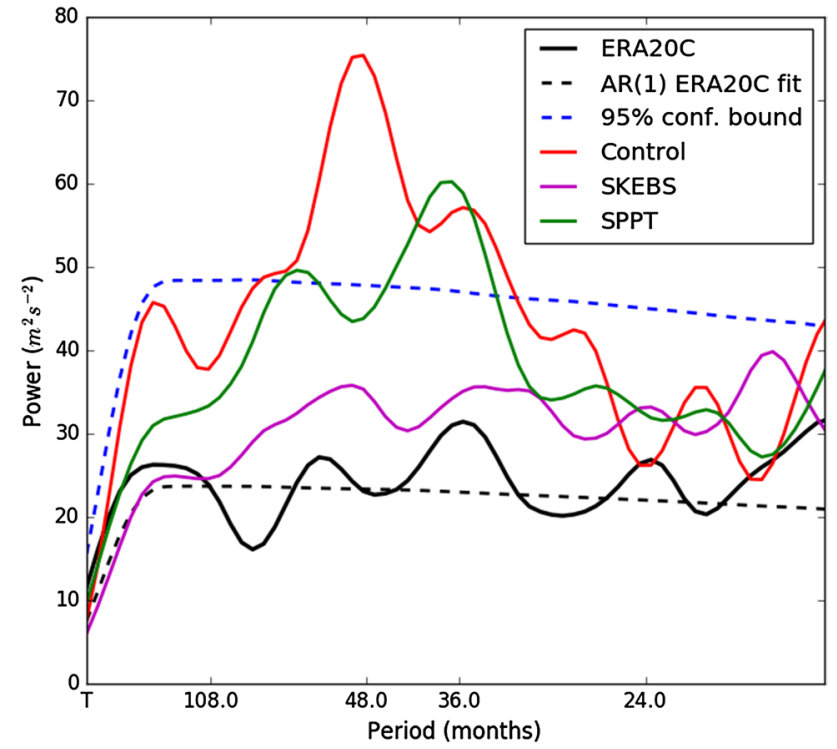

Fig. 9 IOD power spectrum over the years 1900-2000 (smoothed using a Hanning window with width 14). The confidence bound is relative to the AR(1) fit of ERA20C. Power falling within this interval is considered statistically comparable to ERA20C

compared to observations. ${ }^{7}$ Significance testing is done in the same way as for the WY-index, with the observational DMI modelled as an AR(1) process, and indicates no statistically significant difference between observations and SKEBS.

\subsection{Impact on precipitation}

We briefly consider the impact on total precipitation. The spatial biases are shown in Fig. 10, along with the mean absolute error of each model run compared to observations, as measured by $E_{a b s}$.

The bias Control-GPCP can be seen to share features of the U850 bias. In particular, there is more rain along the U850 overturning circulation. This is consistent with the fact that this circulation was enhanced in Control, as this is likely to lead to more water-vapor being advected along this track, enhancing precipitation. The impacts of SKEBS and SPPT are again consistent with the changes in U850, including the increases in mean absolute error.

Figure 11 shows the power spectrum of total precipitation $(\mathrm{mm})$, averaged over the monsoon region, for the years 1900-2000. The spectrum is remarkably similar to that of the WY-index, with the excessive double peaks in Control, and the complementary improvements

\footnotetext{
${ }^{7}$ It is possible that a peak of this height is in fact within the range of observational uncertainty in a manner not captured by our choice of significance testing.
} 
Fig. 10 Means of total precipitation (mm) during monsoon period, averaged over the years 1979-2010: a GPCP, b ControlGPCP, $\mathbf{c}$ SKEBS-Control and d SPPT-Control. $E_{a b s}$ measures the mean absolute error of each scheme (Control, SKEBS and SPPT) compared to observations (a)

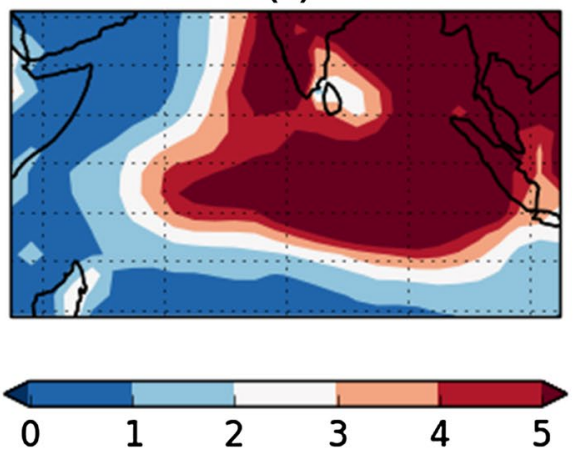

(c) $\left(E_{a b s}=2.42\right)$

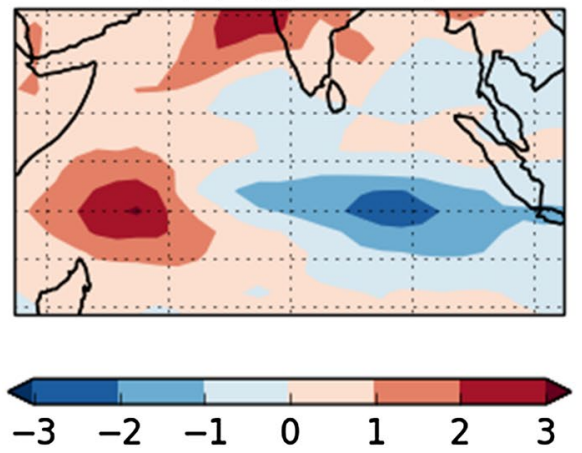

(b) $\left(E_{a b s}=1.90\right)$

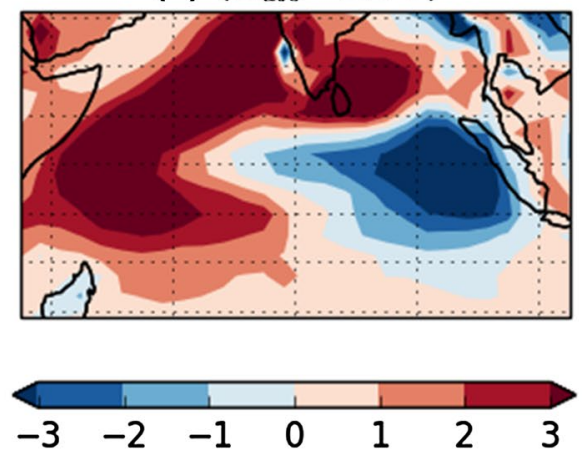

(d) $\left(E_{a b s}=2.04\right)$

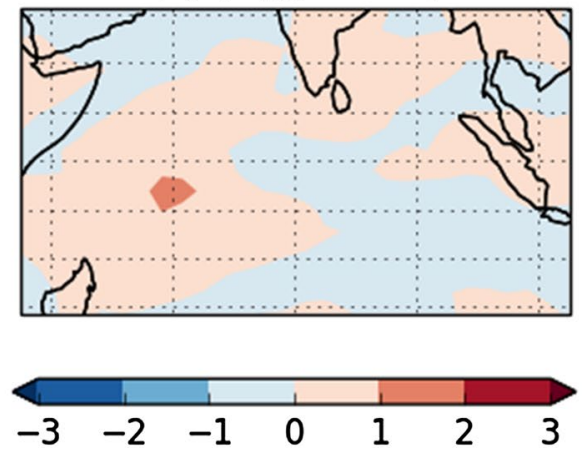

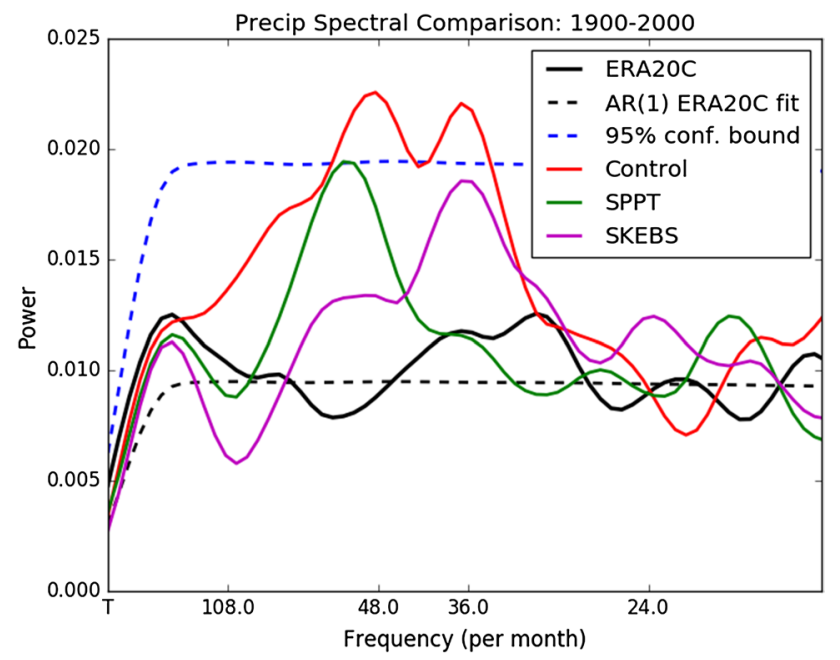

Fig. 11 Total precipitation power spectrum in monsoon region over the years 1900-2000 (smoothed using a Hanning window with width 14). The confidence bound is relative to the AR(1) fit of ERA20C. Power falling within this interval is considered statistically comparable to ERA20C

of SKEBS and SPPT. As a result, we will from now on restrict our attention to the WY-index alone. Entirely analogous results are found when considering precipitation instead.
Table 2 Correlations between the Nino 3.4 timeseries (N34) (yearly averages), IOD index timeseries (SON averages) and Webster-Yang index timeseries (monsoon period averages) over the period 19002000

\begin{tabular}{lrrrr}
\hline & \multicolumn{1}{l}{ ERA-20C } & \multicolumn{1}{l}{ CTRL } & \multicolumn{1}{l}{ SKEBS } & \multicolumn{1}{l}{ SPPT } \\
\hline WY-N34 & $-0.55( \pm 0.07)$ & $-0.46( \pm 0.08)$ & $-0.51( \pm 0.07)$ & $-0.35( \pm 0.09)$ \\
WY-IOD & $-0.50( \pm 0.07)$ & $-0.49( \pm 0.07)$ & $-0.39( \pm 0.08)$ & $-0.37( \pm 0.09)$ \\
IOD-N34 & $0.59( \pm 0.07)$ & $0.72( \pm 0.05)$ & $0.49( \pm 0.07)$ & $0.58( \pm 0.06)$ \\
\hline
\end{tabular}

WY-N34 denotes the correlation between WY and N34, WY-IOD the correlation between WY and IOD and IOD-N34 the correlation between IOD and N34. Standard errors of correlation are shown in parentheses

\section{Discussion}

\subsection{Dependency of WY on ENSO and the IOD}

We saw above that both stochastic schemes were able to improve interannual variability in the monsoon. In order to determine the extent to which these improvements are driven by improvements in ENSO and the IOD, we need to first assess their influence on the monsoon in the model. As first a measure of this influence, we computed the correlation coefficients between the WY-index and the Nino 3.4 
(a)

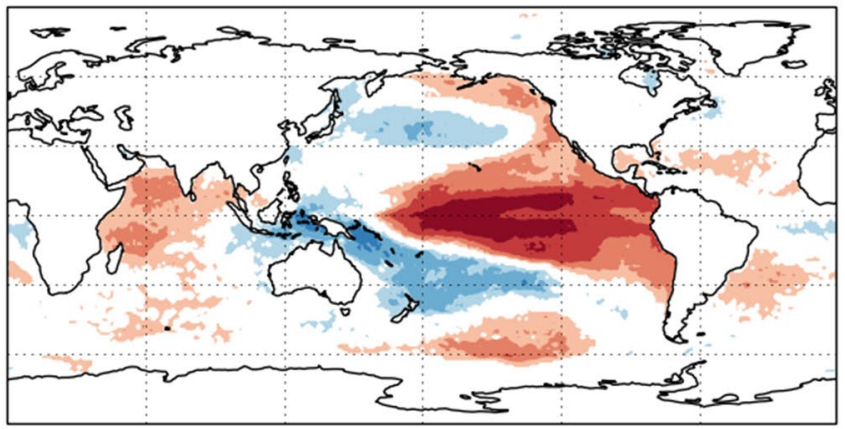

(c)

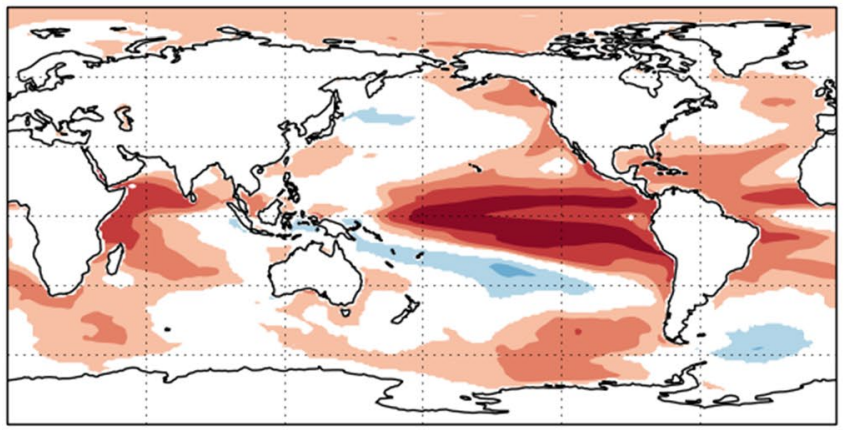

(b)

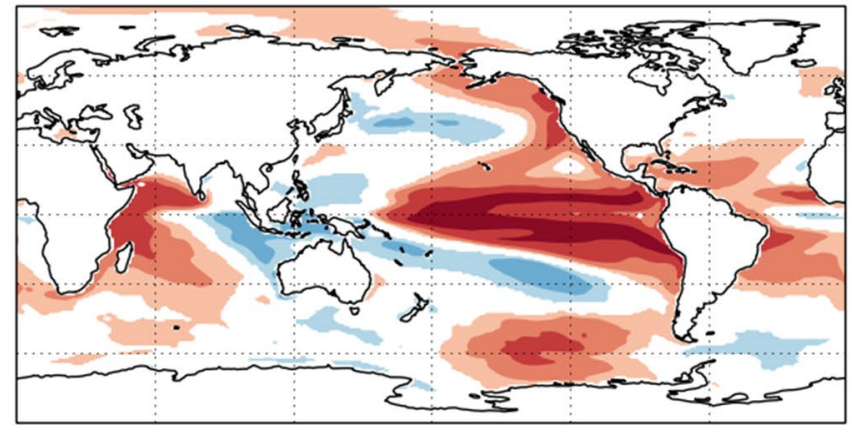

(d)

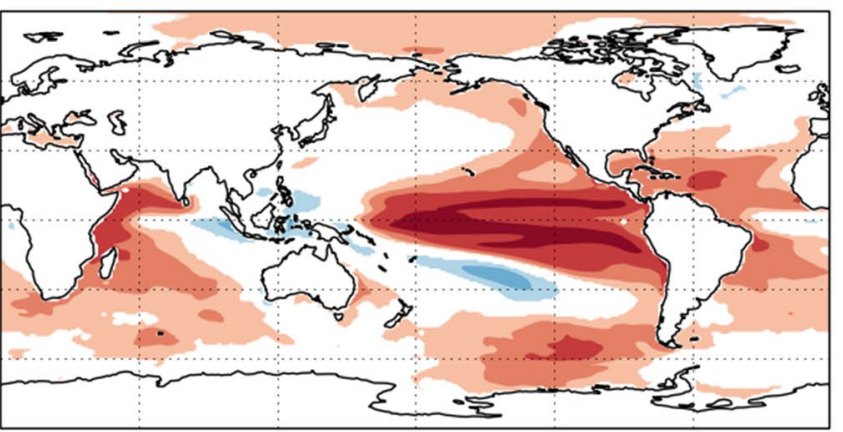

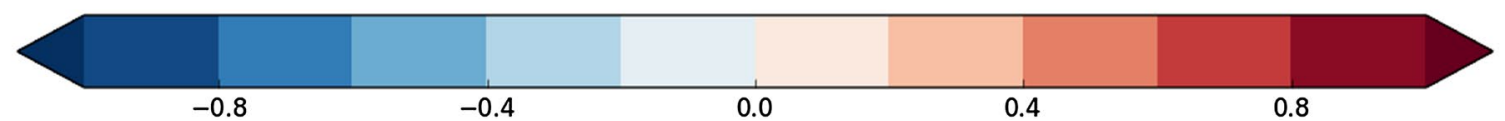

Fig. 12 Correlation coefficients between the yearly Nino 3.4. average and the monsoon period SST mean at different gridpoints, averaged over the period 1979-2010: a ERA-Interim, b Control, c SKEBS and d SPPT. Only statistically significant $(p<0.05)$ correlations are displayed

timeseries (averaged over each year) ${ }^{8}$ and the IOD index averaged over the SON season each year. The results are presented in Table 2, along with the correlations between yearly Nino 3.4 and IOD indices. All timeseries were considered over the period 1900-2000, and all results were found to be statistically significant with respect to the 99th percentile $(p<0.01)$.

We see that the correlation between the WY-index and ENSO is a little bit too weak in CTRL, while the correlation between ENSO and the IOD is overly strong. In SPPT, all correlations have been notably reduced, indicating that the added stochasticity has a strong decoupling effect on these three processes. SKEBS has slightly improved the correlation between WY and N34 towards that in observations, while significantly reducing both correlations related to the IOD. The impact on the IOD correlations can be

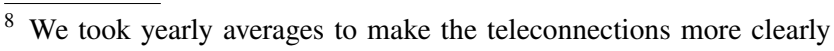
visible.
}

understood further by considering Fig. 12, which shows correlation between the yearly averaged N34 index and monsoon period SST at other gridpoints for each of the models.

The strong dipole pattern visible in CTRL corresponds to the characteristic dipole pattern of the IOD. However, with SPPT, the correlation in the eastern pole has reduced considerably, while for SKEBS it has become statistically insignificant: this explains the way in which SKEBS and SPPT reduced the correlation, with SKEBS reducing it the most.

Based on the data presented so far, we hypothesize that the improved monsoon variability due to SPPT (SKEBS) is explained primarily by its improved representation of the ENSO (IOD). Indeed, the strong connection between SSTs and wind-shear is well known (see Gill 1980; Webster 1972), and the ENSO-induced signal and IOD are the two leading EOFs of Indian Ocean SSTs (Yamagata et al. 2004), so such a causality is not unreasonable to expect. We will test this hypothesis in the next section. 
Table 3 Linear model parameters $\sigma, \mu$ and $\lambda$ estimated by linear regression

\begin{tabular}{lcccr}
\hline & ERA20C & CTRL & SKEBS & SPPT \\
\hline$\sigma$ & 0.93 & 0.92 & 0.89 & 0.91 \\
$\lambda$ & -1.57 & -2.2 & -1.97 & -1.87 \\
$\mu$ & -0.37 & -0.82 & -0.29 & -1.12 \\
\hline
\end{tabular}

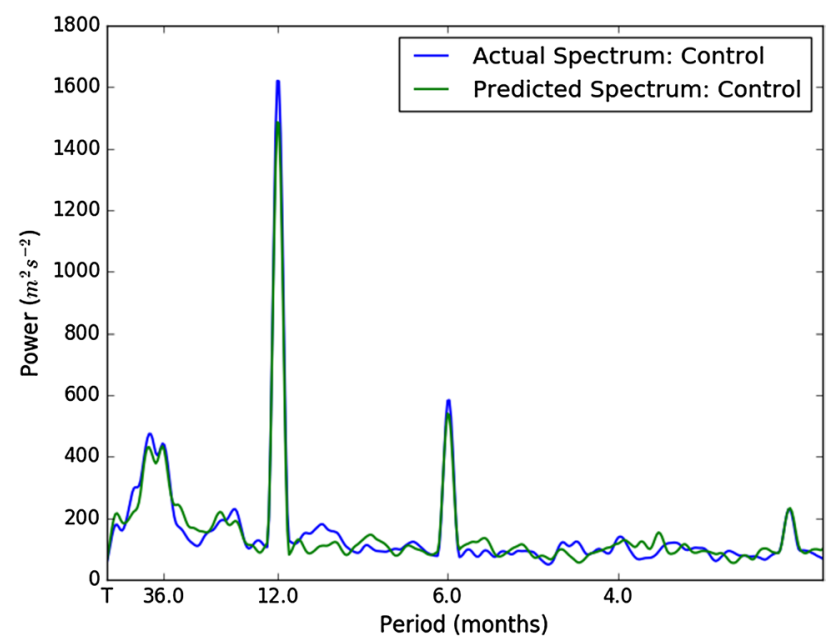

Fig. 13 Actual power spectrum of the monthly WY-index in the deterministic CCSM4 model, along with a typical incarnation of the spectrum predicted by the linear model. Both averaged over the period 1900-2000 and smoothed using a Hanning window of width 14. The seasonal cycle has been kept in this figure, and is responsible for the large peak at 12 months, with harmonics at 6 and 2 months

\subsection{A simple model of the WY-index}

The hypothesis to be tested is that in the CCSM4, the spectral peaks at interannual frequencies of the Webster-Yang index can be attributed almost exclusively to the influence of the ENSO and the IOD. If this is the case, then improvements in these spectral peaks due to SPPT and SKEBS can be attributed almost exclusively to their improvement of these two phenomena. To test this, we will attempt to model the monthly WY-index using the following simplified, linear model:

$\mathrm{WY}(t)=\sigma \mathrm{WY}_{c}(t)+\lambda \mathrm{N} 34(t)+\mu \mathrm{DMI}(t)+\epsilon(t)$.

Here N34 is the Nino 3.4 series, DMI the dipole mode index, $\mathrm{WY}_{c}$ is the series which simply outputs the climatological value of $W Y(t)$ at each month, and $\epsilon(t)$ is a noise term assumed to be normally distributed with mean 0 . In other words, we imagine that modulo random noise, the WY-index is made up of a completely periodic baseline $\mathrm{WY}_{c}$, plus some interannual variability induced by ENSO and the IOD alone. ${ }^{9}$ The parameters $\lambda$ and $\mu$ measure the extent to which the WY-index is driven by ENSO and the IOD respectively: due to the anti-correlation between these phenomena and the monsoon, we expect these to be negative. The factor $\sigma$ is a measure of the periodicity of the monsoon and should be expected to be close to 1 .

We will use multiple linear regression to fit this linear model to each dataset. Table 3 shows the estimated coefficients produced in this way. In each case, the coefficient of determination of the fit was approximately 0.85 (where a score of 1 indicates a perfect fit and a score of 0 would correspond to climatology): this implies that the correlation between the linear model and the data is around 0.9, indicating the very high skill of the linear fit. Figure 13 shows a typical incarnation of the fitted spectrum for Control: its very close match to the observed spectrum supports our hypothesis that in the deterministic model, monsoon variability is driven almost entirely by ENSO and the IOD.

In general, the coefficients are consistent with what we expected, including the fact that the $\mu$ factor for SKEBS is dramatically reduced, in line with the reduced IOD amplitude observed in that run: consistently weaker IODs lead to an on-average reduced coupling between the IOD and the monsoon. For SPPT, the coefficients are broadly comparable to those of Control, indicating that the main cause of improvement is due to reduced periodicity in the ENSO of SPPT, as expected. For SKEBS, the improvement is both due to the reduced amplitude of the IOD but also to due to reduced periodicity.

A detailed analysis of why SPPT improved ENSO was carried out in Christensen et al. (2016): we refer readers to that paper for the details. We propose that the main reason SKEBS has improved the IOD is because it has broken the overly strong correlation between ENSO and the IOD. This allows the IOD to develop more independently, producing a more realistic range of amplitudes and less periodic gaps between events. SPPT has reduced the correlation as well, in line with that of observations. The fact that it is unable to impact on the amplitude of the IOD and retains a too large spectral peak suggests that despite having a realistic ENSO-IOD coupling in terms of consistency, its strength is still too strong. This can be seen in Fig. 12, where the correlation in the western IOD region in particular is too strong in all three model runs. As Control and SPPT also have significant correlation in the majority of the eastern IOD region, ENSO has a strong modulating effect on the IOD amplitude in these runs, while SKEBS elimination of correlation here reduces this effect considerably. So why has SKEBS broken this strong ENSO-coupling?

\footnotetext{
$\overline{9}$ Since $\mathrm{WY}_{c}$ is periodic with period 12 months, it cannot contribute any power at lower, interannual frequencies.
} 
The explanation may stem from the fact that SKEBS has introduced a systematic heating bias. Indeed, the average SST of the deterministic (SPPT) run over 1900-2000 is $290.46 \mathrm{~K}(290.47 \mathrm{~K})$, while for SKEBS it is $291.11 \mathrm{~K}$, an increase of $0.65^{\circ}$. A detailed analysis of the causes of this are beyond the scope of the paper, but is likely to be linked to a systematic reduction in cloud cover induced by the stochastic perturbations. Taking this heating as given, the decrease in correlation with ENSO is now consistent with the results of Zheng et al. (2010), which finds that under a temperature increase, the IOD becomes more independent of ENSO. Specifically, they find that the thermocline in the eastern region shoals under a warming. As a result, it becomes easier for local wind anomalies to trigger up or downwelling in the eastern pole off the coast of Sumatra. Since this up or downwelling is crucial to the development of IOD events (Yamagata et al. 2004), this means that the IOD is no longer as dependent on remotely forced thermocline changes triggered by ENSO, thereby reducing the correlation between the DMI and N34.

It is notable that while SPPT did not introduce a warm bias, it also failed to significantly improve the IOD, suggesting perhaps that the model cannot easily simulate a realistic IOD without having a too warm ocean. We would also note that in the case of SKEBS, the major bias was purely with regards to temperature; there are no major shifts in the mean of other crucial variables likely to be relevant for these results, such as winds or precipitation.

\section{Conclusions}

We investigated the capability of a climate model, CCSM4, to represent observed interannual monsoon variability in simulations with and without stochastic parameterizations. The power spectrum of the WY-index appears to be closely related to ENSO and the IOD, both in observations and in the model, so the impact on these processes was investigated as well. A simple linear model was formulated to quantify the relative impact of ENSO and IOD on the WY-index power spectrum. It was found that in the deterministic model, the coupling of ENSO to the monsoon was too strong. Indeed, interannual monsoon variability, as measured by the power spectrum of both the WY-index and a proxy for All India Rainfall, appears in the model to be driven almost entirely by ENSO and the IOD: ENSO works as the primary driver of the IOD, whence both tend to develop in phase to strongly regulate monsoon intensity. As the ENSO spectrum has too much power in the 3-4 year frequency band in the deterministic model, this leads to the same being the case for the WY-index. Both SPPT and SKEBS notably improved this, reducing the power in this range towards that in observations (though both still show somewhat excessive power).

In the case of SPPT, we linked this, using the linear model, to its improvement of ENSO, as explored in Christensen et al. (2016). For SKEBS, we linked the improvement to an improved representation of the IOD, both in terms of amplitude and variability. We believe the main cause of this is due to SKEBS significant weakening of the ENSO-IOD correlation, especially in the eastern Indian Ocean, allowing the IOD to develop more independently and therefore more realistically. As SKEBS was found to introduce a systematic warming bias in the oceans, this provides further evidence that a shoaling of the eastern thermocline in the Indian Ocean allows the IOD to act more independently of ENSO, as in Zheng et al. (2010). Comparison of our metrics over the first and second half of the century appear to support this, with the improvements from SKEBS being slightly greater in the second half when the temperature has increased more. This suggests that focusing on a more realistic Indian Ocean thermocline may be a route to improving the IOD and the monsoon in future model development. The temperature bias introduced by SKEBS is not fully understood yet and will be examined more closely in the future; it is not clear to what extent the results here are model-specific and whether or not the impact of SKEBS would be similar in another model.

Given the complementary improvements of each scheme, it would also be of interest to perform experiments with both schemes running in tandem. This will be examined further in future studies.

Acknowledgements K.S. acknowledges funding from the European Commission under Grant Agreement 641727 of the Horizon 2020 research programme. The research of H.M.C. and T.N.P. was supported by European Research Council Grant number 291406. The research of J.B. was supported by the US Environmental Protection Agency, Grant number G2011-STAR-D-183520501.

Open Access This article is distributed under the terms of the Creative Commons Attribution 4.0 International License (http:// creativecommons.org/licenses/by/4.0/), which permits unrestricted use, distribution, and reproduction in any medium, provided you give appropriate credit to the original author(s) and the source, provide a link to the Creative Commons license, and indicate if changes were made.

\section{Appendix: Assessing the impact of drift}

In Sect. 2.1 we showed that the majority of model drift for SKEBS and SPPT, as measured by TOA fluxes, had stabilized after the first 30 years. However, the strong change in mean SST's in the SKEBS simulation naturally prompts the question as to whether some of the impact seen is a result of a small amount of remaining drift. To assess the 


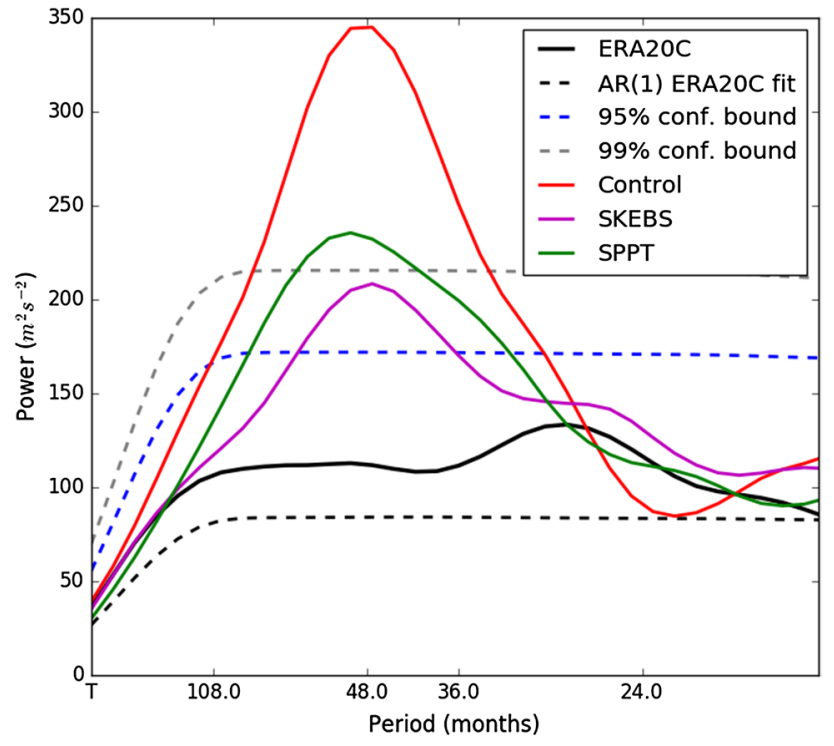

Fig. 14 Power spectrum of monthly WY-index over the years 19001950 (smoothed using a Hanning window with width 14). The confidence bounds are relative to the AR(1) fit of ERA20C

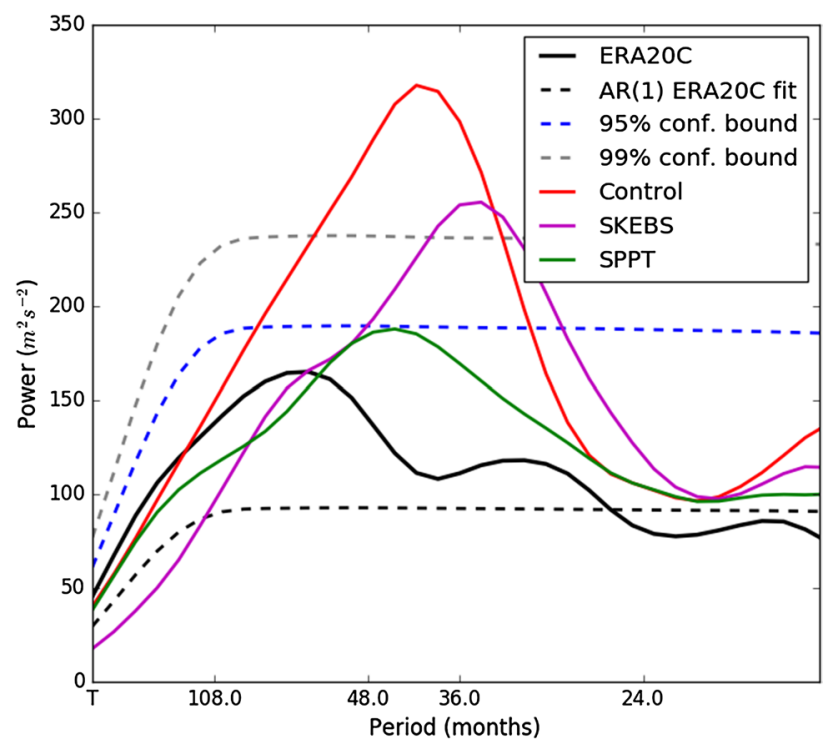

Fig. 15 Power spectrum of monthly WY-index over the years 19502000 (smoothed using a Hanning window with width 14). The confidence bounds are relative to the AR(1) fit of ERA20C

potential effect of this on our results, we repeated our analysis over the two periods 1900-1950 and 1950-2000 to see how the impact changes with time.

Figures 14 and 15 show the power spectrum of the WYindex over these two halves of the 20th century. While there is naturally some variation in time, the change is small when compared to the statistical significance curves for ERA-20C. Control still has an excessive peak, though it appears to shift from 4 to 3 years. SPPT and SKEBS both improve on this, bringing the spectrum within or only just over the $99 \%$ confidence bound, as was the case when considering the entire timeseries. Indeed, if we produce confidence bounds for SPPT and SKEBS based on the entire timeseries, then the variations within the two timeperiods falls well within the $95 \%$ confidence bound of each (not pictured). This suggests that the overall impact on the monsoon is, statistically speaking, roughly constant over the entire time-period and therefore that model drift is not playing a big role.

Furthermore, the difference in globally averaged SST's between Control and SKEBS for the two halves of the 20th century is quite small: in the first half it is around 0.6 compared to 0.68 in the second. In turn, there is a small change in the correlation between ENSO and the IOD in the SKEBS run, starting off at 0.5 in the first half and decreasing slightly to 0.47 in the second. We also see a slight improvement of the IOD power spectrum in the second half, bringing SKEBS even closer to observations (not shown). This is consistent with our hypothesis that the increased Indian Ocean SST's in the SKEBS simulation is a leading cause of IOD improvements. The fact that these slight improvements over time do not translate to a slight improvement in the WY-index is likely because small changes in the IOD are drowned out by natural variability in its own internal ENSO. Indeed, SKEBS seems to have a slightly stronger ENSO in the second half of the century (not shown).

There is no appreciable difference in SPPT spectra or correlations over the two timeperiods, and in terms of drift, its globally averaged SST's differ from Control by around 0.01 over both the first and second half.

\section{References}

Adler RF et al (2003) The version 2 global precipitation climatology project (GPCP) monthly precipitation analysis (1979-present). J Hydrometeorol 4:1147-1167

Annamalai H (2007) IPCC climate models and the Asian summer monsoon. IPRC Clim 7:10-14

Ashok K, Chan WL, Tatsuo M, Yamagata T (2004) Decadal variability of the Indian Ocean Dipole. Geophys Res Lett 31(24):L24207. doi:10.1029/2004GL021345

Ashok K, Guan Z, Yamagata T (2001) Impact of the Indian Ocean Dipole on the relationship between the Indian Monsoon Rainfall and ENSO. Geophys Res Lett 28(23):4499-4502

Berner J, Doblas-Reyes F, Palmer T, Shutts G, Weisheimer A (2008) Impact of a quasi-stochastic cellular automaton back-scatter scheme on the systematic error and seasonal prediction skill of a global climate model. Philos Trans R Soc A 366:2561-2579

Berner J, Shutts G, Leutbecher M, Palmer T (2009) A spectral stochastic kinetic energy backscatter scheme and its impact on flowdependent predictability in the ECMWF ensemble prediction system. J Atmos Sci 66:603-626 
Christensen HM, Berner J, Coleman D (2016) Stochastic parametrisation and the El Niño Southern oscillation. Accepted for publication

Dee D et al (2011) The ERA-interim reanalysis: configuration and performance of the data assimilation system. Q J R Meteorol Soc 137(656):553-597

Gent et al (2011) The community climate system model version 4. J Clim 24:4973-4991

Gill A (1980) Some simple solutions for heat induced tropical circulations. Q J R Meterol Soc 106:447-462

Ju J, Slingo J (1995) The Asian summer monsoon and ENSO. Q J R Meterol Soc 121:1133-1168

Kang IS, Jin K, Wang B, Lau KM, Shukla J, Krishnamurthy V, Schubert SD, Waliser DE, Stern W, Kitoh A, Meehl G, Kanamitsu M, Galin V, Satyan V, Park CK, Liu Y (2002) Intercomparison of the climatological variation of asian summer monsoon precipitation simulated by $10 \mathrm{gcms}$. Clim Dyn 19:383-395

Krishna Kumar K, Rajagopalan B, Cane M (1999) On the weakening relationship between the Indian Monsoon and ENSO. Science 284:2156-2159

Krishna Kumar K, Rajagopalan B, Hoerlin M, Bates G, Cane M (2006) Unravelling the mystery of Indian monsoon failure during El niño. Science 314:115-119

Mason P, Thomson D (1992) Stochastic backscatter in large-eddy simulations of boundary layers. J Fluid Mech 242:51-78

Meyers G, McIntosh P, Pigot L, Pook M (2006) The years of El Niño, La Niña, and interactions with the tropical Indian ocean. J Clim 20:2872-2880

Palmer T, Buizza R et al (2009) Stochastic parametrization and model uncertainty. Tech. Rep. 598. European Centre for MediumRange-Weather Forecasts

Poli P et al (2016) ERA-20C: an atmospheric reanalysis of the 20th century. J Clim 29:4083-4097

Saji N, Goswami BN, Vinayachandran P, Yamagata T (1999) A dipole mode in the tropical Indian Ocean. Nature 401:360-363
Shutts GJ (2005) A kinetic energy backscatter algorithm for use in ensemble prediction systems. Q J R Meteorol Soc 612:3079-3102

Sperber K, Annamalai H et al (2013) The Asian summer monsoon: an intercomparison of CMIP5 vs. CMIP3 simulations of the late 20th century. Clim Dyn. doi:10.1007/s00382-012-1607-6

Sperber K, Palmer T (1996) Interannual tropical rainfall variability in general circulation model simulations associated with the atmospheric model intercomparison project. J Clim 9:2727-2750

Trenberth KE, Fasullo JT, Kiehl J (2009) Earth's global energy budget. Bull Am Meteorol Soc 90:311-324

Walker G (1923) Correlation in seasonal variations of weather, iii: a preliminary study of world weather. Mem Indian Meteorol Dept $24: 275-332$

Webster P (1972) Response of the tropical atmosphere to local, steady, forcing. Mon Weather Rev 100:518-540

Webster P, Yang S (1992) Monsoon and ENSO: selective interactive systems. Q J R Meteorol Soc 118:877-926

Weisheimer A, Corti S, Palmer T, Vitart F (2014) Addressing model error through atmospheric stochastic physical parameterisations: impact on the coupled ECMWF seasonal forecasting system. Philos Trans R Soc A 372:20130290. doi:10.1098/ rsta.2013.0290

Weller E, Cai W (2013) Realism of the Indian Ocean Dipole in the CMIP5 models: the implications for climate projections. J Clim 26:6649-6659

Williams P (2012) Climatic impacts of stochastic fluctuations in airsea fluxes. Geophys Res Lett. doi:10.1029/2012GL051813

Yamagata $\mathrm{T}$ et al (2004) Coupled ocean-atmosphere variability in the tropical Indian ocean. In: Wang C, Xie SP, Carton JA (eds) Earth's climate. American Geophysical Union, Washington, DC. doi:10.1029/147GM12

Zheng XT et al (2010) Indian Ocean Dipole response to global warming: analysis of ocean-atmospheric feedbacks in a coupled model. J Clim 23:1240-1253 